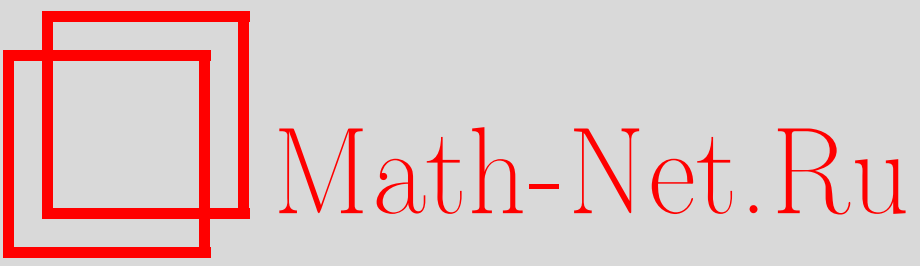

А. В. Пухликов, Бирационально жесткие многообразия с пучком гиперповерхностей Фано, УМH, 1999, том 54, выпуск 1, 261-262

DOI: https://doi.org/10.4213/rm127

Использование Общероссийского математического портала Math-Net.Ru подразумевает, что вы прочитали и согласны с пользовательским соглашением

http://www.mathnet.ru/rus/agreement

Параметры загрузки:

IP : 54.198 .55 .26

26 апреля 2023 г., 16:26:51 


\title{
БИРАЦИОНАЛЬНО ЖЕСТКИЕ МНОГООБРАЗИЯ С ПУЧКОМ ГИПЕРПОВЕРХНОСТЕЙ ФАНО
}

\author{
А. В. Пухликов
}

1. Мы доказываем бирациональную жесткость общих фано-расслоений $\pi: V \rightarrow \mathbb{P}^{1}$, каждый слой которых есть гиперповерхность $W_{M} \subset \mathbb{P}^{M}$ степени $M \geqslant 5$. Для $M=3,4$ этот факт был установлен в [1]. Доказательство проводится методом максимальных особенностей, начало которому положила работа В.А. Исковских и Ю.И. Манина [2], с использованием новых идей, введенных в [1], [3]. Основной результат настоящей статьи может рассматриваться как частный случай общего принципа: если Фано-расслоение $\pi: V \rightarrow S$ "достаточно закручено" по базе, то бирациональная геометрия многообразия $V$ сводится к бирациональной геометрии общего слоя $F_{\eta}$-многообразия Фано над незамкнутым полем $\mathbb{C}(S)$. Возможно более полная реализация этого принципа определяет одно из основных направлений в современной бирациональной геометрии.

2. Опишем явную конструкцию многообразий с пучком гиперповерхностей Фано. Пусть $\mathscr{E}-$ локально-свободный пучок ранга $M+1$ на проективной прямой $\mathbb{P}^{1}, \mathscr{E}=\bigoplus_{i=0}^{M} \mathscr{O}_{\mathbb{P}^{1}}\left(a_{i}\right), a_{0}=$ $0 \leqslant a_{1} \leqslant \cdots \leqslant a_{M}, X=\mathbb{P}(\mathscr{E})$ - соответствующее проективное расслоение. Символом $\mathbb{P}$ будем обозначать проективное пространство $\mathbb{P}^{M}$ - слой канонической проекции $\pi: X \rightarrow \mathbb{P}^{1}$. Пусть $L \in$ Pic $X$ - класс тавтологического пучка и $G \in \operatorname{Pic} X-$ класс слоя $\cong \mathbb{P}$. Для произвольного $m \in \mathbb{Z}$ определим семейство $\mathscr{V}(m)$ подмногообразий коразмерности 1 в $X$ как подмножество линейной системы $|M L+m G|$, состоящее из гладких дивизоров. Здесь параметр $m$ определяет "степень закрученности" Фано-расслоения $V \in \mathscr{V}(m)$ над базой $\mathbb{P}^{1}$. Проекцию $V$ на $\mathbb{P}^{1}$ обозначим тем же символом $\pi$. Общий слой $F_{\eta}$ Фано-расслоения $\pi: V \rightarrow \mathbb{P}^{1}$ - гиперповерхность Фано индекса 1 в $\mathbb{P}_{\mathbb{C}(t)}^{M}$, где $t$ - линейный параметр на прямой. При $m \geqslant 1$ по теореме Лефшеца Pic $V=\left.\operatorname{Pic} X\right|_{V}$, так что Pic $V=\mathbb{Z} K_{V} \oplus \mathbb{Z} F$, где $F=\left.G\right|_{V}-$ класс слоя. Конкретный слой $\pi^{-1}(t)$ над точкой $t \in \mathbb{P}^{1}$ будет обозначаться символом $F_{t}$.

Очевидно, $K_{V}=-L_{V}+\left(a_{1}+\cdots+a_{M}-2+m\right) F$, так что чем больше $m$, тем "меньше" антиканоническая линейная система $\left|-K_{V}\right|$.

3. Теперь введем точные условия "закрученности" $V$ по базе и одно необходимое нам условие общности положения. Пусть $g \in A^{2}(V)$ - класс гиперплоского сечения слоя $F_{t} \subset \mathbb{P}$. Фано-расслоение $\pi: V \rightarrow \mathbb{P}^{1}$ удовлетворяет $K^{2}$-условию, если численный класс циклов коразмерности 2 на $V N K_{V}^{2}-g$ не является эффективным для любого $N \in \mathbb{Z}$. Поскольку линейная система $\left|L_{V}\right|$ свободна, $K^{2}$-условие выполнено для $V$, если справедливо неравенство $\left(K_{V}^{2}\right.$. $\left.L_{V}^{M-2}\right) \leqslant 0$. Последний индекс пересечения, как нетрудно подсчитать, равен $M\left(4-a_{1}-\cdots-\right.$ $\left.a_{M}\right)-m(2 M-1)$. Поэтому неравенство $m \geqslant \frac{M}{2 M-1}\left(4-\sum_{i=1}^{M} a_{i}\right)$ гарантирует выполнение $K^{2}$-условия.

Рассмотрим произволную гиперповерхность $W \subset \mathbb{P}$ степени $M$. Пусть $p \in W, z_{*}=$ $\left(z_{1}, \ldots, z_{M}\right)$ - система линейных координат на $\mathbb{P}$ с центром в точке $p, q=q_{1}+q_{2}+\cdots+q_{M}$ - уравнение гиперповерхности $W$ относительно координат $z_{*}$, разложенное на однородные компоненты $q_{i}$ степени $i$.

Точка $p \in W$ называется регулярной неособой точкой гиперповерхности $W$, если последовательность $\left(q_{1}, \ldots, q_{M-1}\right)$ регулярна. Точка $p$ называется регулярной особой точкой гиперповерхности $W$, если $q_{1} \equiv 0$, последовательность $\left(q_{2}, \ldots, q_{M}\right)$ регулярна, квадрика $q_{2}=0$ в $\mathbb{P}^{M-1}=T_{p} \mathbb{P}$ неособа и, более того, система уравнений $q_{2}=\cdots=q_{M}=0$ определяет в $\mathbb{P}^{M-1}=T_{p} \mathbb{P}$ конечное множество, состоящее из ровно $M$ ! различных точек, любые $M$ из которых линейно независимы.

Фано-расслоение $\pi: V \rightarrow \mathbb{P}^{1}$ назовем регулярныцм, если любой его слой $F_{t}$ - $\mathbb{Q}$-факториальное многообразие (при этом группа классов дивизоров Вейля, тензорно умноженная на $\mathbb{Q}$, изоморфна $\mathbb{Z}$ и порождена гиперплоским сечением $\left.F_{t} \subset \mathbb{P}\right)$, любая точка которого $p \in F_{t}$ регулярна.

Работа выполнена при поддержке Российского фонда фундаментальных исследований (грант № 99-15-96013). 
Можно показать, что достаточно общее многообразие $V$ есть регулярное Фано-расслоение.

Определение бирациональной (сверx) жесткости и другие необходимые определения см. в $[1],[3]$.

Основной результат настоящей работы -

Теорема. Пусть $\pi: V \rightarrow \mathbb{P}^{1}$ - Фано-расслоение, построенное выше. Предположим, что оно регулярно и удовлетворяет $K^{2}$-условию. Тогда оно бирационально сверхжесткое.

СледСтвия. (i) Любое бирациональное отображсене $\chi: V \rightarrow W$ на Фано-расслоение $\rho: W \rightarrow S$ является послойным, т.е. $S=\mathbb{P}^{1}$ или точка, и в первом случае существует изоморфизм $\alpha: \mathbb{P}^{1} \rightarrow S$ такой, что $\alpha \circ \pi=\rho \circ \chi$. (ii) Группа бирациональньих автоморфизмов многообразия $V$ совпадает с группой его бирегулярных автоморфизмов: $\operatorname{Bir} V=$ Aut $V$. Для общего члена семейства $\mathcal{V}(m)$ эта группа тривиальна. (iii) Многообразие $V$ не рационально.

4. Опишем теперь схему доказательста теоремы. Зафиксируем $\chi: V \rightarrow W$, где $\rho: W \rightarrow S-$ $\Phi$ ано расслоение, и предположим, что для некоторой подвижной системы $\left|D^{\prime}\right|$ на $W$ условие монотонности для порогов канонического присоединения не выполнено: $n=c(D, V)>c\left(D^{\prime}, W\right) \geqslant 0$. Наша цель - привести это предположение к противоречию. Линейная система $|\chi|=|D|$, $D \sim-n K_{V}+l F, l \geqslant 0$, не имеет неподвижных компонент. Напомним, что геометрическое дискретное нормирование $\nu \in \mathcal{N}(V)$ называется максимальной особенностью, если оно удовлетворяет неравенству Нетера-Фано $(-$ Манина-Исковских) $e(\nu)=\nu(|\chi|)-n a(\nu, V)>0$, где $a(\cdot)$ - дискрепантность нормирования относительно заданной модели. Пусть $D_{i} \in|\chi|-$ общие дивизоры, $Z=\left(D_{1} \bullet D_{2}\right)$ - алгебраический цикл их теоретико-схемного пересечения, $Z=Z^{v}+Z^{h}$ - его разложение на вертикальную (т.е. содержащуюся в слоях) и горизонтальную (т.е. накрываюшую базу $\mathbb{P}^{1}$ ) компоненты, соответственно. Для $Z^{v}$ имеем дальнейшее разложение $Z^{v}=\sum_{t \in \mathbb{P}^{1}} Z_{t}^{v}, \operatorname{Supp} Z_{t}^{v} \subset F_{t}$. Можно показать [1], что существует максимальная особенность $\nu \in \mathscr{M}$, центр которой лежит в слое, $\operatorname{centre}(\nu) \subset F=F_{t}$, такая, что $e=e(\nu)>\frac{\nu(F)}{2 M n} \operatorname{deg} Z_{t}^{v}$. Мы назовем ее сверхмаксимальной [1] и зафиксируем.

5. Главная часть доказательства - исключение сверхмаксимальной особенности. Это делается с помощью техники подсчета кратностей в форме [1], которая восходит к технике пробного класса В.А. Исковских и Ю. И. Манина [2]. Самый трудньй случай - когда центр сверхмаксимальной особенности есть особая точка $p \in F$ слоя. Здесь противоречие достигается с помощью следующего ключевого факта. Пусть $\sigma: \widetilde{F} \rightarrow F$ - раздутие особенности $p, E \subset \widetilde{F}$ - исключительный дивизор (неособая квадрика), $x \in E$ - некоторая точка. Пусть $Y \subset F$ - простой дивизор Вейля, $\widetilde{Y} \subset \widetilde{F}$ - его собственный прообраз. Тогда

$$
\frac{\operatorname{mult}_{x} \tilde{Y}}{\operatorname{deg} Y} \leqslant \frac{2}{M} .
$$

Предположение о существовании максимальной особенности приводит к противоречию. Значит, условие монотонности для порогов канонического присоединения выполнено, а это и означает бирациональную сверхжесткость. Полный текст доказательства см. в [4].

\section{СПИСОК ЛИТЕРАТУРЫ}

[1] Пухликов А. В. // Изв. РАН. Сер. матем. 1998. Т. 62. №1. С. 123-164. [2] Исковских В. А., Манин Ю. И. // Матем. сб. 1971. Т. 86. №1. С. 140-166. [3] Pukhlikov A. V. // Invent. Math. 1998. V. 134. № 2. P. 401-426. [4] Pukhlikov A. V. Birationally rigid Fano fibrations // MPI-Preprint 1998-113. 